\title{
Climatic Change
}

\section{A healthier US diet could reduce greenhouse gas emissions from both the food and health care systems \\ --Manuscript Draft--}

\begin{tabular}{|c|c|c|}
\hline Manuscript Number: & \multicolumn{2}{|l|}{ CLIM-D-15-00517R1 } \\
\hline Full Title: & \multicolumn{2}{|c|}{$\begin{array}{l}\text { A healthier US diet could reduce greenhouse gas emissions from both the food and } \\
\text { health care systems }\end{array}$} \\
\hline Article Type: & \multicolumn{2}{|l|}{ Research Article } \\
\hline Corresponding Author: & \multicolumn{2}{|l|}{$\begin{array}{l}\text { David A Cleveland, PhD } \\
\text { University of California Santa Barbara } \\
\text { Santa Barbara, CA UNITED STATES }\end{array}$} \\
\hline \multicolumn{3}{|l|}{$\begin{array}{l}\text { Corresponding Author Secondary } \\
\text { Information: }\end{array}$} \\
\hline Corresponding Author's Institution: & \multicolumn{2}{|l|}{ University of California Santa Barbara } \\
\hline \multicolumn{3}{|l|}{$\begin{array}{l}\text { Corresponding Author's Secondary } \\
\text { Institution: }\end{array}$} \\
\hline First Author: & \multicolumn{2}{|l|}{ Elinor Hallström, PhD } \\
\hline \multicolumn{3}{|l|}{ First Author Secondary Information: } \\
\hline \multirow[t]{4}{*}{ Order of Authors: } & \multicolumn{2}{|l|}{ Elinor Hallström, PhD } \\
\hline & \multicolumn{2}{|l|}{ Quentin Gee, PhD } \\
\hline & \multicolumn{2}{|l|}{ Peter Scarborough, PhD } \\
\hline & \multicolumn{2}{|l|}{ David A Cleveland, PhD } \\
\hline \multicolumn{3}{|c|}{ Order of Authors Secondary Information: } \\
\hline \multirow[t]{2}{*}{ Funding Information: } & Energimyndigheten (SE) & Dr Elinor Hallström \\
\hline & $\begin{array}{l}\text { Royal Swedish Academy of Agriculture } \\
\text { and Forestry }\end{array}$ & Dr Elinor Hallström \\
\hline Abstract: & \multicolumn{2}{|c|}{$\begin{array}{l}\text { The standard US diet contributes to greenhouse gas emissions (GHGE) from both the } \\
\text { food system, and from the health system through its contribution to noncommunicable } \\
\text { diseases. To estimate the potential for diet change to reduce GHGE and improve } \\
\text { public health, we analyzed the effect of adopting healthier model diets in the US on the } \\
\text { risk of disease, health care costs, and GHGE. We found that adoption of healthier diets } \\
\text { reduced the relative risk of coronary heart disease, colorectal cancer, and type } 2 \\
\text { diabetes by } 20-45 \% \text {, US health care costs by US } \$ B \text { B } 54-72 \text { per year, and GHGE by } \\
133-1618 \mathrm{~kg} \text { capita-1 yr- } 1 \text { ( } 65-106 \mathrm{~kg} \text { from the health care system, } 68-1512 \mathrm{~kg} \text { from } \\
\text { the food system). Using the more realistictimely } 20 \text {-year global warming potential for } \\
\text { methane increased emissions substantially, especially from the food system. Emission } \\
\text { reductions were equivalent to } 4-37 \% \text { of the US Climate Action Plan's target of a } 17 \% \\
\text { reduction in } 2005 \text { GHGE by } 2020 \text {, and } 26-150 \% \text { of California's target of } 1990 \mathrm{GHGE} \\
\text { levels by } 2020 \text {. Given the urgency of improving public health and of mitigating GHGE } \\
\text { over the short term, diet change could play a much more prominent role in health and } \\
\text { climate policies. Our results add to knowledge of the positive synergistic effects of diet } \\
\text { change that could help motivate those changes, both at the individual and policy levels. }\end{array}$} \\
\hline Response to Reviewers: & & \\
\hline
\end{tabular}


1 A healthier US diet could reduce greenhouse gas emissions from both the food and health care

2 systems

4 Electronic supplementary material The online version of this article contains supplementary

5 material, Online Resource 1, in which we provide detailed information on methods, including

6 sources of data for GHGE and RR, and for results and discussion.

81 Introduction

10 There is increasing scientific consensus that significant reductions in anthropogenic greenhouse

11 gas emissions (GHGE) will have to be achieved in the next decade or two to limit global

12 warming to $2^{\circ} \mathrm{C}$ to avoid dangerous climate change (Hansen et al. 2013; IPCC 2014; Rogelj et al.

13 2013; Stocker 2013). Our individual and collective diets, and the food system that creates and

14 supports them, contribute about one third of total emissions globally (Garnett 2011), and

15 changing dietary patterns can reduce GHGE embodied in the diet by up to 50\% (Hallström

16 2014). However, compared with other mitigation strategies the food system has received

17 relatively little attention (Edenhofer 2014).

18 Diet change is an attractive mitigation option as it requires no new technologies, minimal

19 new infrastructure, and has important positive externalities, including improved health. In

20 addition, because agricultural production is the largest contributor of anthropogenic methane

$21\left(\mathrm{CH}_{4}\right)$, for example 34\% of total US $\mathrm{CH}_{4}$ emissions (calculated with data in EPA 2014b), which

22 has a short life span in the atmosphere and a 20-year global warming potential (GWP) 86 times 
1 that of $\mathrm{CO}_{2}$ (IPCC 2013), diet change could make an important contribution to reducing GHGE

2 over the critical short term.

3 Diet change also has the potential to reduce GHGE from the health care system by

4 improving health. The average intake of foods by the US population, the standard American diet

5 (SAD), has become markedly less healthy in recent decades, and in combination with an

6 increasingly sedentary lifestyle, has resulted in an epidemic of non-communicable diseases

7 (NCD) (Grotto and Zied 2010). About half of all US adults have one or more NCDs, and about

8 two-thirds are overweight or obese (USDA and HHS 2015); the prevalence of diabetes was 9\%

9 in 2012 (CDC 2012; CDC 2014), the prevalence of cardiovascular disease (including high blood

10 pressure and various forms of coronary heart disease) in the population over 20 years old in 2010

11 was $35 \%$ (Go et al. 2014), and $40 \%$ of the population is predicted to be diagnosed with cancer

12 during their lifetime (SEER NCI 2014). NCDs are important contributors to increasing U.S.

13 health care costs, which were almost $\$ 3$ trillion $\mathrm{yr}^{-1}$ in $2014,18 \%$ of the total US GDP (CMS

14 2013). NCDs can to a large extent be prevented by adopting a healthy lifestyle, including healthy

15 diets (WCRF/AICR 2007; WHO/FAO 2003).

16 The link between diet, health, and climate is a new but growing research field. By using

17 life cycle assessments (LCAs) (ISO 2006a; ISO 2006b), the climate impact of individual foods, 18 meals and whole diets have been calculated (Scarborough et al. 2014a; Tilman and Clark 2014).

19 Health aspects have been included in LCAs by expressing the climate impact of the food with a

20 functional unit that relates to the nutritional content (Doran-Browne et al. 2015), or by

21 comparing the climate impact of diets with varying nutritional content (Meier and Christen

22 2012; Saxe et al. 2013; van Dooren et al. 2014; Westhoek et al. 2014). So far, few studies have

23 used epidemiological data to study the relationship between diets' impact on both GHGE via the 
1 food system, and on health (Aston et al. 2012; Friel et al. 2009; Scarborough et al. 2012;

2 Springmann et al. 2016; Tilman and Clark 2014). The results from these studies show that

3 dietary change offers substantial potential to simultaneously improve health and reduce GHGE

4 from the food system.

5 However, there is no research to date on the combined effect on GHGE from the food

6 and health care systems of the adoption of more healthy diets. Here we report research that

7 addresses this gap. We modeled counterfactual healthy alternative diets (HADs) by adjusting

8 foods in the SAD which are statistically associated with changes in the relative risk for three

9 NCDs. We then estimated the change in GHGE from both the food and health care systems. Our

10 results provide new insights into the relationship between food, health and climate that have

11 important policy implications in the development of more sustainable food systems and the

12 mitigation of anthropogenic climate change.

13

14

152 Methods

16

17 Additional information is available in Online Resource 1.

18

19

202.1 System boundaries

21

22 The spatial boundary of our study was the US, although we extrapolated LCA data for the food

23 system from other countries when US data were not available, and data for disease risk were 
1 from various countries. The reference year for our study was 2013, and we used either data for

2 2013, extrapolated data to 2013 based on trends, or used data for the closest year when there was

3 no basis for extrapolation. The system boundaries for emissions from the health care system

4 (GHGE-H) were the components of the health care sector associated with the studied NCDs. The

5 system boundaries for emissions from the food system (GHGE-F) were inputs to production at

6 the distal end, including feed and pasture for animals, through retail at the proximal end, because

7 these were the boundaries for the LCA studies we used, and included food wasted within these

8 boundaries. Thus GHGE-F from land use change, and for retail to consumer transport, storage

9 and preparation at the consumer stages, and household food waste disposal were not included,

10 making our estimates conservative. GHGE (in terms of $\mathrm{CO}_{2} \mathrm{e}$ ) comprised the three major

11 greenhouse gases, $\mathrm{CO}_{2}, \mathrm{~N}_{2} \mathrm{O}$, and $\mathrm{CH}_{4}$.

142.2 Overview of methodology

16 Our method for estimating the effect of dietary change on GHGE comprised six steps (Fig. 1). In

17 step 1, we defined the SAD using data on loss-adjusted food availability at the consumer level,

18 identified foods linked to change in relative risk (RR) for NCDs, and created the HADs by

19 adjusting these foods in the SAD. In step 2, we estimated the changes in disease prevalence with

20 HADs, based on RR estimates found in published meta-analyses. In step 3, we estimated

21 changes in health care expenditures from dietary change, based on changes in disease prevalence

22 from step 2 . In step 4, we estimated $\triangle$ GHGE-H from dietary change, based on the cost of health

23 care for each NCD from step 3. In step 5, we estimated $\triangle$ GHGE-F using LCA data found in the 
1 literature. In step 6 we estimated the net total change in GHGE from dietary change, $\Delta$ GHGE-T

$2=\Delta \mathrm{GHGE}-\mathrm{F}+\Delta \mathrm{GHGE}-\mathrm{H}$.

4 Fig. 1 HERE

$7 \quad 2.3$ Step 1. Developing dietary scenarios

9 We used the SAD as our reference and compared it with three counterfactual healthy alternative 10 diets (HAD-1, -2, -3) (Table 1). Dietary intake levels in SAD are based on the USDA per capita 11 loss-adjusted food availability data by weight for 2012 , the most recent year for which data were 12 available (USDA ERS 2014).

13 To create the HADs, we adjusted SAD only for foods which met our three criteria: (i)

14 USDA dietary recommendations were consistent with international dietary guidelines and

15 recommendations (USDA and HHS 2010; WCRF/AICR 2007), (ii) there were high quality data

16 correlating them with disease, and (iii) there were documented GHGE estimates from LCAs. The

17 changes in HADs were increased consumption of fruits, vegetables, whole grains, and beans and

18 peas, and reduced consumption of refined grains, and red (beef and pork) and processed

19 (preserved by smoking, curing or salting, or addition of chemical preservatives) meat. Creation

20 of the HADs thus involved only a portion of the total SAD. We did not change any other food

21 groups (e.g. sugar sweetened beverages, white meat, fish, dairy, eggs) because they did not meet

22 one or more of our three criteria. For example, we did not find enough high quality data linking

23 added sugar or sugar sweetened beverages with CHD, T2D, or CRC. 
HAD-1 reflects the USDA dietary recommendations, the "USDA Food Pattern" (USDA

2 and HHS 2010), and processed meat is limited to $20 \%$ of total red meat. In HAD-2 and HAD-3

3 red and processed meat were further reduced to $25 \mathrm{~g}$ of cooked meat day ${ }^{-1}$ in HAD-2, to zero in

4 HAD-3, and replaced by increases in beans and peas. The replacement of meat with plant-based

5 protein is based on a framework developed by the USDA (USDA 2010) (see Online Information, 6 section 1.1).

8 Table 1 HERE

112.4 Step 2. Changes in relative risk of disease with changes in diet

12

13 We based the selection of diseases to be included on a literature review of the NCBI Pub Med

14 database in March 2014, using as keywords the selected food groups (e.g. "vegetables") and

15 CHD, hypertension, type 2 diabetes (T2D) and different types of cancer. We selected peer

16 reviewed meta-analyses of prospective cohort studies, randomized controlled trial studies, and

17 case-control studies, published between 2005 and 2014, that provided RR with 95\% confidence 18 intervals $(\mathrm{CI})$.

19 We estimated the health effects of changing the diet from SAD to HADs by calculating a 20 revised RR $\left(R R_{r e}\right)$ (Eq. 1) for each food-disease RR (Scarborough et al. 2014b), as reported in the 21 meta-analyses, proportional to the changes of that food in the HADs: 
2 where $R R$ is the original RR obtained from meta-analyses for food $f$ (e.g., processed meat) and

3 disease $d$ (e.g., CHD), $x$ is the level of $f$ in the HAD, $y$ is the level of $f$ in the SAD, and $u$ is the

4 unit increase reported in the meta-analysis identified for disease $d$.

$5 \quad$ We then calculated the combined relative risk $\left(R R_{c d}\right)$ of the changes in all of the foods

6 contributing to the RR for each disease by multiplying them:

10 where $R R_{r e 1}, R R_{r e 2}, R R_{r e 3}$, and $R R_{r e f}$ are the revised $\mathrm{RR}$ values for each of the individual foods 11 changed in the diet.

12 Finally, to construct $95 \%$ confidence intervals around the relative risk estimates for the 13 HADs, we conducted a Monte Carlo simulation (Rubinstein 2007) with 5000 iterations in which 14 the individual RR estimates for each disease were allowed to vary randomly according to a 15 lognormal distribution. The result of a Monte Carlo simulation consists of a number of possible 16 outcomes of the calculation, hence giving a representation of the probability of different results 17 depending on the uncertainty and variation in the input data.

212.5 Step 3. Changes in health care costs from changes in disease prevalence 
1 In calculating the reductions in health care costs due to reductions in the RR of the three NCDs

2 for each of the three HADS, we assumed that reduction in these costs in the US economy for

3 each disease were directly proportional to reduction in the $R R_{c d}$ for each disease. We used the

4 most recent and reliable data sources for expenses, and adjusted for inflation.

$7 \quad 2.6$ Step 4. Change in GHGE due to changes in health care costs ( $\triangle G H G E-H)$

9 We calculated per capita reduction in GHGE-H for the US population based on established 10 relationships between different types of health care costs and their GHGE in the US using data 11 from the Carnegie-Mellon IO-LCA (GDI 2014). The IO-LCA GHGEs are based on older IPCC 12 estimates of GWP, and we also calculated GHGE by adjusting the GWP for $\mathrm{CH}_{4}$ from 21 to the 13 most recent IPCC estimates (including climate-carbon feedbacks) of 34 for a 100-year time 14 frame, and 86 for a 20-year time frame\{Myhre, 2013 \#12366@714, Table 8.7\} (see Online 15 Information section 1.6). While the GWP for $\mathrm{N}_{2} \mathrm{O}$ changed in the most recent IPCC assessment, 16 the difference was much smaller (from 310 to 298 for the 100-year time frame), and would also 17 have been more difficult to adjust.

202.7 Step 5. Changes in GHGE in the food system ( $\triangle$ GHGE-F)

22 We quantified the effect on GHGE-F from changes in diet based on estimates of the GHGE of 23 the specific foods included in the diets, provided from a review of published LCAs. The 
1 literature search was performed in Web of Knowledge (ISI) in April 2014, using as keywords the

2 foods included in the studied diets and LCA. We also manually searched reference lists from

3 retrieved articles and reviews. GHGE-F data were collected for 28 different food categories and

4 the median, lowest, and highest amount of GHGE-F kg${ }^{-1}$ food available at the retail were

5 determined (Tables OR 2, 3, 4). We adjusted the GHGE-F data to match the system boundaries

6 of this study and to reflect the US food system, e.g. by adjusting the proportion of air transported

7 fruit and hot house produced vegetables. GHGE-F data were for conventional food production

8 and data on food produced outside of the US were limited to regions with similar production

9 systems as the US and to regions that export food to the US.

10 In order to address uncertainty and variability in our GHGE-F results due to differences

11 in production systems, regional conditions and methods used in different studies (Björklund

12 2002; Nijdam et al. 2012; Röös and Josefine 2013), we conducted a Monte Carlo simulation

13 (Rubinstein 2007). Because of the limited number and range of estimates in the literature, we

14 used a triangular distribution model for each food category changed in the HADs from the

15 baseline SAD. Each diet iteration involved a summation of the random values within each food

16 category's distribution. The 5000-iteration Monte Carlo simulation then produced a best estimate

17 and confidence intervals for GHGE-F for each diet.

18 For beef, we also estimated GHGE based on different GWPs for methane. We did not

19 adjust the GWP for methane from the other food categories since emissions data were often not 20 broken out by greenhouse gas, which makes our estimates conservative. 
12.8 Step 6. Net change in GHGE from dietary change ( $\triangle G H G E-T)$

2

3 The net change in GHGE from dietary change was calculated as the sum of emission reductions

4 in the food $(\triangle \mathrm{GHGE}-\mathrm{F})$ and the health care systems $(\triangle \mathrm{GHGE}-\mathrm{H})$.

$7 \quad 3$ Results and discussion

9 We found that our HADs resulted in large decreases in GHGE in the health care system, but 10 especially in the food system, and that these could make major contributions to US and 11 California climate change mitigation goals. Our estimates are conservative because the HADs 12 included only a selection foods, a selection of associated NCDs, a selection of direct health care 13 GHGE of those conditions, and did not include some important components of food system 14 GHGE (e.g. from land use change and the consumer level).

\subsection{Reduction in relative risk of disease and health care costs}

19 The combined RR $\left(R R_{c d}\right)$ for CHD, T2D and CRC for all foods changed in the HADs was 20 reduced by $20-45 \%$, with the largest reduction for CHD, followed by T2D and CRC (Table 21 OR5). HAD-3, in which all red and processed meat was replaced with legumes, provided the 22 greatest reduction in disease prevalence of the HADs. 
The potential annual savings in US health care costs with reduction in prevalence of

2 CHD, T2D and CRC, assuming that the entire US population made a transition from the SAD to

3 the HADs, was $\$ 54, \$ 65$ and $\$ 72$ billion $\mathrm{yr}^{-1}$ for HAD-1, -2 , and -3 , or $20-30 \%$ of the total

4 expenses for these disease of \$219.5 billion $\mathrm{yr}^{-1}$ (CMS 2013).

73.2 Reduction in GHGE

9 Reduction in GHGE-H (Table 2) was estimated from the reduction in health care expenses 10 resulting from the reduction in RR (Table OR5), and thus shows a similar pattern to RR.

12 Table 2 HERE

14 Reductions in GHGE-F were strongly affected by the incremental reduction in red and

15 processed meat (Table 1), since they account for a large proportion of GHGE and of methane, 16 resulting in reductions from $6 \%$ to $70 \%$ (Table 3 ). The relatively small emission reduction

17 potential for HAD-1 was due to increased emissions from the large increment in fruits and 18 vegetables relative to the reduction in red and processed meat (Table 1). Greater reductions in 19 GHGE were achieved by HAD-2 and -3 due to additional reductions in red and processed meat, 20 with no further increase in fruits and vegetables. 
2 for methane (see Online Information section 1.6). Many studies continue to use older IPCC

3 estimates of the 100-year GWP of methane (21 in 1996, 23 in 2001, 25 in 2007), while the 2013

4 estimate is 34. GHGE-F for SAD increased by $17 \%$ when 100-year GWP values for methane

5 were updated to the current estimate, although only including methane from beef. While the

6 IPCC states that the choice of one GWP time horizon over another is a value judgment with no

7 scientific basis (IPCC 2013), most LCAs use the 100-year GWP. However, since $\mathrm{CH}_{4}$ has a

8 lifespan of 12.4 years (IPCC 2013), using a 20-year GWP more accurately reflects its climate

9 impact over the critical short term (Howarth 2014), especially for foods with high $\mathrm{CH}_{4}$

10 emissions, like beef and other ruminant foods. $\mathrm{CH}_{4}$ absorbs high amounts of radiation in its short

11 lifespan, resulting in a much higher value for the 20-year time frame. Using the 20-year GWP for

$12 \mathrm{CH}_{4}$ doubled the GHGE reductions for the HADs compared with the 100-year GWP of 34.

13 Fig. 2 shows the combined reduction in GHGE capita ${ }^{-1} \mathrm{yr}^{-1}$ with a transition from the

14 SAD to the HADs and how these are affected by using different GWP values for methane.

15 Reductions in GHGE-F dominated reduction potential in all HADs, accounted for 51-93\% of

16 total potential, and increased with increasing $\mathrm{CH}_{4} \mathrm{GWP}$ values (Table OR6). The total including

17 GHGE-H, the mitigation potential increased a maximum of about 90\% for HAD-1 using a GWP

18 for $\mathrm{CH}_{4}$ of 21, but decreased with increasing GWP for $\mathrm{CH}_{4}$, and for HAD-2 and -3 because of

19 the greater contribution of $\mathrm{CH}_{4}$ to GHGE-F. For HAD-3 using a GWP for $\mathrm{CH}_{4}$ of 86 , including

20 GHGE-H only increased the total by $7 \%$. 
$2 \quad 3.3$ Limitations and uncertainty

4 The results of this study are dependent on the quality of data used for food consumption, food

5 losses, RR, health care expenses, and GHGE. In order to minimize, account for and illustrate the

6 overall uncertainty in our results, we used validated data of high quality, aimed for high

7 transparency in presenting our methods, and estimated the uncertainty of both GHGE-F and RR

8 of food-related diseases with Monte Carlo simulation.

\section{$11 \quad 3.3 .1 \mathrm{GHGE}-\mathrm{H}$}

13 A major assumption in our GHGE-H calculations was that the changes in RR are directly related

14 to health care costs. In reality, diet change would only affect disease prevalence over time via

15 reduction in incidence. Our results should therefore be interpreted as theoretical estimates of the

16 disease prevalence attributable to the HADs over time, or as the health care costs associated with

17 a counterfactual scenario where the HADs had always been adopted.

18 To minimize the risk of double counting health effects when combining RR estimates, we 19 only used RR estimates from meta-analyses that adjusted for influencing confounders, such as 20 other types of food intake, physical activity level, and history of disease. Also, by using whole 21 food-based recommendations (e.g. vegetables), as opposed to nutrient-based recommendations

22 (e.g. fiber), we reduced the risk of double counting health effects from nutrients found in various 23 food groups. Despite these efforts, some risk of double counting remains, because the RRs are 
1 drawn from meta-analyses of mainly prospective cohort studies which have measured usual diet

2 imprecisely, allowing for residual confounding. For increased transparency, we presented the

3 health effects of the HADs both for each dietary factor individually (Table OR 1) as well as for

4 the combined effect of all dietary changes (Table OR5).

5 There are also some uncertainties in the IO-LCA data used to calculate GHGE-H because

6 it uses an aggregate-based assignment of GHGE for economic activity in a given industry/sector,

7 as opposed to process/product based assignment. Some components of the LCA, such as

8 pharmaceutical manufacturing, leave out potentially important factors that could add to the

9 GHGE of a given health care expenditure, and some components of our calculations were based

10 on proxy data because GHGE data were not available for all activities. While it is not clear that

11 the health care sector would experience the same rate of decrease in carbon intensity as the

12 overall economy, we did adjust it to match this rate.

13 A more fundamental issue in estimating GHGE-H is the increase in life span with

14 reduced risk of NCDs, which would increase the length of time individuals would be generating

15 GHGE from their activities. Longer life expectancies may also result in increases in additional

16 health care expenses for comorbid conditions. On the other hand, the total health care costs

17 might be reduced if many years of care for T2D, CRC, or CHD were replaced by fewer years of

18 less expensive health care, and higher economic productivity. We did not consider the potential

19 rebound effect of health care cost savings being used for equally or more carbon intensive

20 activities, as this is a separate issue. We also did not consider the effect on GHGE of economic

21 adjustments due to changes in demand for different foods (Jensen et al. 2013).

22 Reductions in GHGE-H were underestimated because we did not include many potential 23 diseases (e.g. overweight and obesity, hypertension, stroke, and forms of cancer other than CRC) 
1 associated with the foods we did change in HADs, due to lack of adequate RR documentation. In

2 addition, there are also potential diet-disease links for foods we did not change in HADs, e.g.

3 added sugar. Therefore, the total estimated health care expenses was a small part of the potential

4 contribution of food-related diseases to the total US health care spending in 2013 of $\$ 2.9$ trillion

5 (CMS 2013).

6

$8 \quad 3.3 .2$ GHGE-F

10 A main source of uncertainty in LCA results is the necessity of using data with a range of 11 characteristics from a variety of sources, as we did. This is true both at the global (e.g. Bajželj et 12 al. 2014), and at the regional or national level. Due to lack of data, GHGE data for US foods are 13 typically sourced from other industrial countries with a range of "climatic conditions, 14 transportation distances, and production methods and therefore are intended to provide a 15 reasonable range of expected values, rather than a definitive result, for each food type" (Heller 16 and Keoleian 2015). Life cycle data on GHGE-F related to the same food item can vary 17 significantly according to different sources due to differences in production systems, regional 18 conditions and methods used to produce the data (Björklund 2002; Nijdam et al. 2012).

19 Uncertainty was also due to the necessity of using data for a limited selection of foods for each 20 category. For higher precision, regional or country level LCA data would be required which are 21 currently lacking.

22 We addressed this uncertainty in GHGE-F estimates with a Monte Carlo simulation using 23 a triangular distribution model for each food category as discussed above. This method assumes 
1 that uncertainty in GHGE estimates for each food group are independent of each other, which is

2 a reasonable assumption because most data were from different studies.

3 GHGE-F estimates were conservative because we did not include land use changes,

4 which can increase emissions from the diet by 10-30\% (Röös et al. 2015), although this varies

5 greatly depending on the assessment method used.

6 Potential GHGE-F reductions may also have been underestimated because only a portion

7 of the foods in the SAD was changed in the HADs , while other foods with high emissions (e.g.

8 dairy and other meats) were not. For example, one estimate for the GHGE of dairy in the US in

92008 was $133.6 \mathrm{Tg} \mathrm{CO}_{2} \mathrm{e} \mathrm{yr}^{-1}$, (using a 100 year GWP for methane of 25) (Thoma et al. 2013),

10 equivalent to $439 \mathrm{~kg} \mathrm{CO}_{2} \mathrm{e}$ capita $^{-1} \mathrm{yr}^{-1}$, which is $\sim 30 \%$ of the GHGE we estimated for SAD (for 11 a 100 year GWP for methane of 34). Our results are also likely underestimates to the extent that 12 the EPA underestimates livestock methane emissions in the US, which could by almost 50\% 13 (Turner et al. 2015), and points to the need for policies for the livestock industry, the only major 14 sector not required to report GHGE.

173.4 Significance

19 To our knowledge, this is the first study of diet GHGE to: model counter factual diets by making 20 incremental changes based on recommendations only for foods for which there were high quality 21 RR data for noncommunicable diseases; estimate reduction in GHGE-H from the health care 22 sector resulting from a change to these diets, in addition to GHGE-F; and use the current and 23 more timely 20-year GWP for $\mathrm{CH}_{4}$. Our HADs are counterfactual diets illustrating the potential 
1 for foods that decrease the RR of NCDs to also decrease GHGE, and are meant to illustrate the

2 potential for diet change to contribute simultaneously to improving health and mitigating and

3 climate change.

4 Compared with previous estimates of just the GHGE-F embodied in the diets of

5 industrialized countries (most of which use a methane GWP of 21), which are in the range of

6 1400-3200 $\mathrm{kg} \mathrm{CO}_{2} \mathrm{e}_{\text {capita }}{ }^{-1} \mathrm{yr}^{-1}$ (Hallström et al. 2014), HAD-1 to -3 would reduce emissions

$7 \sim 4-44 \%$ (for a CH4 GWP of 21). When using the 20-year CH4 GWP, our results showed that in

8 terms of the total US capita ${ }^{-1}$ GHGE, the maximum potential reduction was less than $3 \%$ with

9 HAD-1, up to 7\% with HAD-3 (Table OR7), which is equivalent to removing $\sim 9$ to 108 million

10 vehicles from US roads (based on $4.75 \mathrm{MT} \mathrm{CO}_{2} \mathrm{e}^{\text {vehicle }}{ }^{-1} \mathrm{yr}^{-1}$ (EPA 2014a).

11 However, the real significance of the potential of HADs is in comparison with mitigation

12 targets. For example, the range of contributions to mitigation targets potentially achievable for

13 HADs (from HAD-1, $\mathrm{CH}_{4} \mathrm{GWP}=21$ to $\mathrm{HAD}-3, \mathrm{CH}_{4} \mathrm{GWP}=86$ ) is $4-37 \%$ for the US

14 President's Climate Action Plan goal of a 17\% reduction below US 2005 net GHGE levels by

152020 , and 27-151\% for California's AB 32 goal of reaching 1990 emission levels by 2020

16 (assuming 2020 emissions equal to 2012 emissions) (Table OR7). This suggests that promoting

17 diet change could be an effective and efficient strategy for reaching near-term GHGE mitigation

18 goals, with relatively minimal investment in new research, technology and infrastructure. 


\section{Conclusions}

3 In accordance with several previous studies (Hallström 2014; Scarborough et al. 2014a; Tilman

4 and Clark 2014; Westhoek et al. 2014) \{Springmann, 2016 \#12035\}, our results support the

5 hypotheses that healthier eating habits can contribute to large reductions in GHGE and disease,

6 and that reduction of animal foods, especially red and processed meat, is key.

7 In addition, we show for the first time that the reduction in health care costs with

8 reduction in NCDs could also reduce GHGE. The potential for this reduction is likely much

9 greater than our estimates, which were conservative, and limited by the lack of data. We also

10 show that using the more timely, short-term GWP of methane can have a major effect on

11 estimating the impact of diet change. This is important, because keeping within the $2^{\circ} \mathrm{C}$ increased

12 temperature limit will likely require reduction in GHGE in the near term, including in the food

13 system (Bajželj et al. 2014; Springmann et al. 2016).

14 Given the urgency of mitigating GHGE and improving public health over the short term,

15 diet change could play a much more prominent role in national, state and local climate policies.

16 Because most food choice decisions are the result of unconscious habit, and differ from

17 perceived barriers to climate friendly food choices, and because the public seems largely

18 unaware of a link between diet and climate change (Mäkiniemi and Vainio 2013), there is a role

19 for increased education about the relationships among food choice, climate and nutrition.

20 Increased knowledge of the positive synergistic health effects of reducing GHGE via diet change

21 could motivate greater efforts in the future to develop and implement such changes, both at the

22 individual and policy levels. Our results provide new knowledge that could help motivate

23 effective change. 


\section{1 \\ 35 Tables}

Table 1 Foods in SAD that were changed in HADs

\begin{tabular}{|c|c|c|c|c|c|c|c|}
\hline \multirow{2}{*}{ Food } & \multicolumn{4}{|c|}{ g capita $^{-1}$ day $^{-1}$ a } & \multicolumn{3}{|c|}{ Change from SAD to } \\
\hline & $\mathrm{SAD}^{\mathrm{b}}$ & HAD-1 & HAD-2 & HAD-3 & HAD-1 & HAD-2 & HAD-3 \\
\hline Total red $\&$ processed meat & 92 & 51 & 25 & 0 & $-45 \%$ & $-73 \%$ & $-100 \%$ \\
\hline Unprocessed red meat & 58 & 41 & 25 & 0 & $-29 \%$ & $-57 \%$ & $-100 \%$ \\
\hline Processed meat $^{d}$ & 34 & 10 & 0 & 0 & $-71 \%$ & $-100 \%$ & $-100 \%$ \\
\hline Total fruits and vegetables & 335 & 672 & 707 & 741 & $101 \%$ & $111 \%$ & $121 \%$ \\
\hline Fruits & 74 & 299 & 299 & 299 & $304 \%$ & $304 \%$ & $304 \%$ \\
\hline Fruit juices & 60 & 75 & 75 & 75 & $25 \%$ & $25 \%$ & $25 \%$ \\
\hline Vegetables without beans & 194 & 283 & 283 & 283 & $46 \%$ & $46 \%$ & $46 \%$ \\
\hline Beans and peas & 7 & 15 & 50 & 84 & $114 \%$ & $614 \%$ & $1100 \%$ \\
\hline Total grains & 167 & 131 & 131 & 131 & $-22 \%$ & $-22 \%$ & $-22 \%$ \\
\hline Whole grains & 17 & 79 & 79 & 79 & $365 \%$ & $365 \%$ & $365 \%$ \\
\hline Refined grains & 150 & 52 & 52 & 52 & $-65 \%$ & $-65 \%$ & $-65 \%$ \\
\hline
\end{tabular}

bSAD based on loss adjusted food availability (USDA ERS 2014).

${ }^{c}$ Red meat refers to beef and pork.

dProcessed meat refers to meat preserved by smoking, curing or salting, or addition of chemical preservatives. 
A healthier US diet could reduce GHGE from food and health care systems, p. 20

Table 2 Reduction in GHGE-H with change to HADs $\left(\mathrm{kg} \mathrm{CO}_{2} \mathrm{e} \text { capita- }{ }^{-1} \mathrm{yr}^{-1}\right)^{\mathrm{a}}$

\begin{tabular}{|c|c|c|c|c|c|c|c|c|c|c|}
\hline \multicolumn{2}{|l|}{ Diet } & \multicolumn{3}{|c|}{ HAD-1 } & \multicolumn{3}{|c|}{ HAD-2 } & \multicolumn{3}{|c|}{ HAD-3 } \\
\hline \multicolumn{2}{|c|}{$\mathrm{CH}_{4} \mathrm{GWP}$} & 21 & 34 & 86 & 21 & 34 & 86 & 21 & 34 & 86 \\
\hline \multirow[t]{4}{*}{ NCD } & CHD & 15 & 16 & 20 & 16 & 18 & 22 & 17 & 18 & 23 \\
\hline & $\mathrm{T} 2 \mathrm{D}$ & 47 & 50 & 63 & 55 & 58 & 73 & 58 & 62 & 78 \\
\hline & CRC & 3 & 3 & 4 & 3 & 4 & 5 & 4 & 4 & 5 \\
\hline & Total & 65 & 69 & 87 & 75 & 80 & 100 & 78 & 84 & 106 \\
\hline
\end{tabular}

relationship (Table OR5), the associated reduction in health care costs, and

the GHGE generated by these costs. 
A healthier US diet could reduce GHGE from food and health care systems, p. 21

Table 3 GHGE-F for different methane GWP for foods changed in HADs, and reductions from SAD

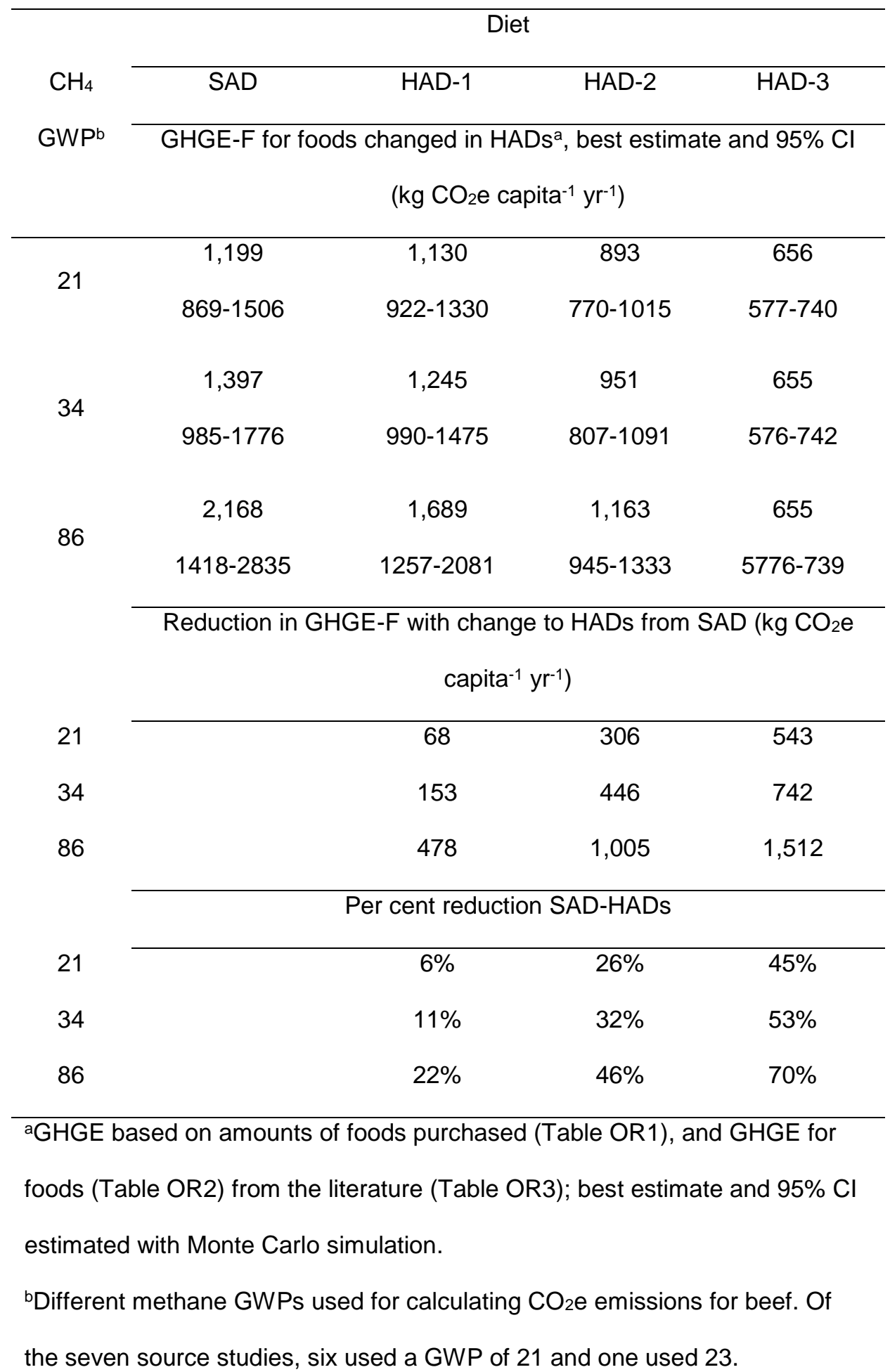


A healthier US diet could reduce GHGE from food and health care systems, p. 22

6

8 
A healthier US diet could reduce GHGE from food and health care systems, p. 23

\section{Figures captions}

2

3 Fig. 1 Flow diagram of research design. RPM=red \& processed meat, $F \& V=$ fruits \& vegetables,

$4 \quad \mathrm{~B} \& \mathrm{P}=$ beans \& peas; see text for other acronyms

5

6 Fig. 2 Reduction in GHGE with HADs from the food and health care systems 


\section{Response to reviewers comments on Ms CLIM-D-15-00517}

Title: A healthier US diet could reduce greenhouse gas emissions from both the food and health care systems

Authors: Elinor Hallström, Quentin Gee, Peter Scarborough, David A. Cleveland

\section{CONTENTS}

1. Reviewer \#1:

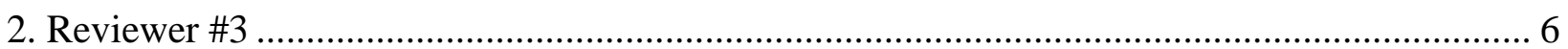

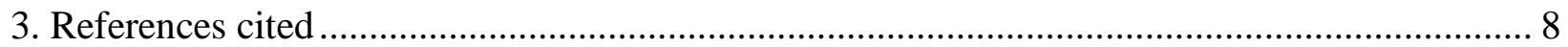

\section{Reviewer \#1:}

Summary of Comments on CLIM-D-15-00517.pdf

1.1. This manuscript seeks to quantify GHGE from selected US diets, including contributions from the food itself and from the associated health care costs. This is a valuable analysis and makes an important contribution to our ability to link food systems, health and environment. As interest increases in quantifying health externalities of our food system, these numbers may get a lot of mileage. I concur with the authors that this is a novel approach. The paper is also very well written, researched and referenced. Given the complexity of doing this work, I appreciated that they included a detailed limitations section.

Major comments

1.2. * In describing the project and results, please qualify the statement that the analysis reflects "total" GHGE. Below are a few places in the text to edit: p5 line 17, p10 line 46, p11 line 19, p17 line 26, Figure 1.

RESPONSE: We have clarified in section 2.1 that "GHGE", also referred to as $\mathrm{CO}_{2} \mathrm{e}$ in our paper, comprises only $\mathrm{CO}_{2}, \mathrm{~N}_{2} \mathrm{O}$ and $\mathrm{CH}_{4}$. The reviewer may have misinterpreted our reference to "net total change in GHGE" as "total GHGE". For the examples cited, our submitted ms. states:

p. $5,1.17$, "net total change in GHGE from dietary change, $\Delta$ GHGE-T $=\Delta$ GHGE-F + $\triangle$ GHGE-H",

p. 10, 1. 46, "Reduction in GHGE-H with change to HAD",

p. 11, 1. [7-] 9, "Greater reductions in GHGE were achieved by HAD-2 and -3",

p. 17, 1. 26, [we see no statement about GHGE here],

Fig. 1, "net total change in GHGE from dietary change, $\Delta$ GHGE-T".

1.3. Should state early on that this is a conservative estimate because it includes only selected foods, selected associated NCDs, selected direct health care GHGE of those conditions, and does not include associated non-health care GHGE.

RESPONSE: We have added a brief summary statement including reasons why our results are conservative at the beginning of section 3 in the main text. 
1.4. * The authors describe a lot of methods, but there are still a number of places where more detail would be helpful in understanding the work, as noted below. Perhaps an appendix would be appropriate if it gets too long.

RESPONSE: In the submitted Online Resource we documented details of the methods, and referenced these in the main text. We have added further clarifications as documented in our other responses to reviewers' comments.

Minor comments and requests for clarification of methods

1.5. * P4 Line 39: Clarify why including only food wasted at the consumer and postconsumer levels. If you used USDA food waste data, I believe that would include retail and restaurant unless you specifically excluded it. (If a change is made here, also change line 31)

RESPONSE: We have clarified in section 2.1 by adding that our boundaries were determined "because these were the boundaries for the LCA studies we used". However, as explained in the submitted ms., because the amount of food used in estimating GHGE was calculated by beginning with amount in our model diets, and adding amount wasted from farm gate to consumer, the GHGE for this wasted food were included, but not the additional GHGE incurred at the consumer level.

We have deleted that confusing sentence (lines 34-41 in the submitted ms.). We have clarified text in section 2.1 that food waste was included within our system boundaries for GHGE-F, which we explained in the submitted ms. (section 2.1: "inputs to production at the distal end, including feed and pasture for animals, through retail at the proximal end"), and which was based on the methods of the LCA studies we used.

We also explained in the Online Resource (section 1.5) the methodology we used to account for food waste in estimating the amount of food purchased (i.e. "retail" amount): "The purchased amount of food in SAD was based on "consumer weight" data from 2012 (USDA ERS 2014) which refer to the weight of the product as it is purchased at the retail level, i.e. after subtracting retail-level losses but before losses at the consumer level (Muth et al. 2011). The amount of food purchased in the HADs was quantified by assuming the same percentage of household food losses in each food category as in the SAD."

1.6. * P5 line 6: explain concept of RR, given the audience

RESPONSE: We added these definitions in the Online Resource (section 1.2): "Meta-analyses used in our study are mainly based on prospective cohort studies in which the RR was (cumulative incidence of the disease in a population with a given level of intake of a food)/(cumulative incidence of the disease in that population with a different level of intake of that food). In case control studies, odds ratios were assumed to approximate RRs. The RR as applied in our scenarios is the estimate of the proportional difference in the risk of disease between an individual consuming the current diet and an individual consuming the dietary scenario." 
We also added this further clarification in the first paragraph of section 2.4: "and case-control studies" (because one of the studies we used as a source of RRs (Micha et al. 2010) used odds ratios from case studies which they "assumed to approximate" RRs.

1.7. * How did you address refined grains in the GHG estimates - was it only the added ghg of refining them compared to whole grain versions?

RESPONSE: We did not differentiate between whole grains and refined grains in the GHG-F calculations as these data were lacking; instead we differentiated among grains between cereals/corn and rice (shown in Table OR2, and OR4), for both whole grains and refined grains, and calculated the GHGE for cereals/corn and for rice separately. A clarification is added to section 1.5 in the Online Resource.

1.8. * Why were SSB and fish not included? I believe these could meet your criteria. Also there could be a way to look at added sugar. I am not asking you to redo the analysis, but put a brief comment at least.

RESPONSE: We explained our choice of foods in section 2.3 of the submitted ms. We edited the first sentence in the second paragraph to make more explicit: "To create the HADs, we adjusted SAD only for foods which met our three criteria". We have also added as an example that added sugar and SSBs were excluded since the evidence of diet-disease correlation was judged insufficient.

1.9. * Clarify how the HAD scenarios match with the dietary changes observed to result in the RRs? If these are extrapolating significantly beyond the dietary changes studied, that would be a limitation to note.

RESPONSE: We added in the second paragraph of section 2.4 a clarification that we calculated revised $\mathrm{RR}\left(R R_{r e}\right)$ for each food-disease $\mathrm{RR}$, as reported in the meta-analyses, "proportional to the changes of that food in the HADs". The methodology used is also described in detail in section 1.2 of the Online Resource. We also clarified, in a paragraph added at the end of that section, that the dietary changes studied are within the range of data in the meta-analyses for the changes in red and processed meat, fruit and vegetables and whole grains.

1.10. * How did you handle it if there were multiple meta analyses?

RESPONSE: When there was more than one meta-analysis $R R_{r e}$ for a food-disease combination, we used an arithmetic average. This is explained in the submitted Online Resource (section 1.2).

1.11. * Note in limitations: the frequency of comorbidities is another reason the calculated health care impacts may be "off" - summing might not get the right answer.

RESPONSE: This comment is difficult to understand, and we hope that we have not misunderstood the reviewer. We think the reviewer is concerned that the saving in health care costs that we have calculated is an over-estimate, since we were not able to allow for additional health care expenses for comorbid conditions as a result of longer life expectancies (due to reductions in NCDs in the scenarios). We acknowledge that this is a limitation and have added a sentence about this in the fourth paragraph of section 3.3.1.

1.12. * What kind of search was performed to identify the LCA studies used? 
RESPONSE: We performed a literature review of previously published LCA data of the different food groups included in the diets studied. We have added clarification of the methods in the first paragraph of section 2.7 in the $\mathrm{ms}$.

1.13. * Reference needed for the proportions of air transported fruit and hothouse vegetables RESPONSE: We cite Wells et al. (for proportion hothouse grown tomatoes and peppers) and Volpe et al. (for proportion air transported) in the submitted Online Resource (section 1.5). We have clarified that text to state that we could find no data on proportion of imported fruits in the US food supply by transport mode, but made conservative estimates based on the limited information in Volpe et al.

1.14. * P11 first paragraph: Not sure if this is a word count issue, but this is a lot of acronyms. Even knowing what they all mean, it's hard to read. This is also an issue with fig 1, among other places. In Fig 1, could consider replacing some of it with visual icons.

RESPONSE: We are already pushing word count limits so prefer not to replace acronyms. We feel that we used a fairly limited amount of acronyms, most of which are standard (e.g. GHGE, IPCC, GWP), and chemical formulae $\left(\mathrm{CO}_{2}, \mathrm{~N}_{2} \mathrm{O}, \mathrm{CH}_{4}\right)$. We do not think it would be an improvement to try to use icons in Fig. 1.

1.15. * P17 line 36, clarify what calculation you're doing here.

RESPONSE: We rewrote this sentence [actually on numbered p. 15, last paragraph in section 3.3 , in the submitted ms.] to clarify that we were illustrating the magnitude of GHGE-F for one of the foods not changed in the HADs: "For example, one estimate for the GHGE of dairy in the US in 2008 was $133.6 \mathrm{Tg} \mathrm{CO}_{2} \mathrm{e} \mathrm{yr}^{-1}$ (using a 100 year GWP for methane of 25) (Thoma et al. 2013), equivalent to $439 \mathrm{~kg} \mathrm{CO}_{2} \mathrm{e}_{\text {capita }}^{-1} \mathrm{yr}^{-1}$, which is $\sim 30 \%$ of the GHGE we estimated for SAD (for a 100 year GWP for methane of 34)."

1.16. * P18 line 21: "may be no more challenging for the case of diet than for other ghg mitigation measures" -- Maybe don't say this unless you want to provide literature or something else to back it up. I'm not convinced it's true. Also it's kind of apples to oranges.

RESPONSE: We deleted the referenced text.

1.17. * P18 line 31: add in update that it wasn't ultimately included in the DGs.

RESPONSE: The paragraph in which this sentence appears (actually p. 17 of the submitted ms., lines 24-36) has been removed because not central to our paper, and to be within word limits.

1.18. * P18 line 45: note that even when they know about it, evidence seems fairly consistent that environment isn't a top motivator for most. (Though this varies - may be more motivating in millenials, for example.)

RESPONSE: See response to 2.8 below.

1.19. * P19 Consider mentioning that the meat industry is the only major GHG emitting industry in the US that is not required to report emissions. Indeed, livestock and other dietrelated GHG are rarely tracked and included in national targets. Given the magnitude of the potential impact you are describing, governments should consider incorporating 
it. This would be a valuable message to put in front of Climatic Change readers, to tune them in to this omission.

RESPONSE: We agree that this is an important issue and have added reference to this at the end of section 3.3.2 in the ms.

1.20. * Table 1: [add into the related text] How were the \% changes determined, esp for had-2 and 3? What was the rationale for the substitutions chosen in reduced meat diets? There are multiple ways of doing this, but it's not clear from the numbers how you approached this.

RESPONSE: The methodology used to create the dietary scenarios is described in detail in section 1.1 in the Online Resource. For clarification we have also added some further description to the main text (section 2.3).

1.21. * Table 1: Why increases in fruit juices? Do the dietary guidelines recommend that? RESPONSE: The USDA food pattern recommends that the total intake of fruit and juices should increase. It is further recommended that "the majority of the fruit intake should come from whole fruits, including fresh, canned, frozen, and dried forms, rather than from juice" (USDA and HHS 2010), but there is no specification of the exact amounts of the intake that should come from whole fruits and juices. Based on this, the total intake of fruit and juices in the HADs was increased according to the USDA guidelines but the proportion of the total intake coming from fruit juices was limited to $20 \%$ (described in the Online Resource, section 1.1., second paragraph). We have also clarified that the juice in the HADs was not sweetened fruit beverages; the USDA considers "sweetened juice products with minimal juice content, such as juice drinks, as sugar-sweetened beverages rather than fruit juice".

1.22. * Table 1: In HADs 1 and 2, it could be reasonable to assume that other meat types would increase if red and processed meat decreased. While the GHGE is not as high in poultry and pork as it is for red meat, it's still higher than most other foods, so this result overstates the impacts of the change.

RESPONSE: In our study red meat refers to both beef and pork, based on the definition by the World Cancer Research Fund, and we have clarified this in the revised ms. (section 2.3 and table 1) and in the Online Resource (section 1.1, paragraph 1). As described in section 2.3 in our submitted ms. (and in the Online Resource, section 1.1), our model assumed that beans and peas substituted for protein in meat, and that there was no change in other foods in the diet as a result of the foods changed in the HADs. It is true that the reduction in GHG would have been smaller if red meat would have been replaced by poultry instead of beans and peas in the HADs; this was however not the scenario we studied.

1.23. * Table 1: How was processed meat defined? (It could be made from multiple different animals and could double-count red meat.)

RESPONSE: Processed meat in our study refers to meat preserved by smoking, curing or salting, or addition of chemical preservatives, based on the definition by the World Cancer Research Fund, this has been clarified in the revised ms. (section 2.3) and in the Online Resource (section 1.1). To avoid double counting the health effect of reducing intake of red meat, a distinction was made between unprocessed and processed red meat when calculating the changes in relative risk (see table OR5 in Online Resource, section 2.1). 


\section{Reviewer \#3}

2.1. Reviewer \#3: The paper analyses the effect on health and greenhouse gas emissions from a changed diet in US. The paper adds to the literature by considering health effect and effect on greenhouse gas emissions simultaneously. The overall method seem valid, even though I cannot evaluate the parameters used for health consequences. Still, I have some major objections to the paper that has to be addressed before publishing.

2.2. The method to estimate the GHG effect due to lower health care expenses is flawed. RESPONSE: We do not agree that the method to estimate health care expenses, and their GHGE, is flawed. Reviewer 3 does not provide any details for this statement, so we cannot address any specific concerns s/he has. We described in the submitted ms. (sections 2.5 and 2.6) and the submitted Online Resource (sections 1.3 and 1.4) our methods for estimating the changes in health care expenses from changes in disease prevalence, and for estimating the changes in GHGE from changes in health care expenses. We were clear about our assumption that the changes in RR followed a log-linear response, and that reductions in RR would have a proportional impact on economic activity. We believe that these methods are more detailed and accurate than those used in much of the previous research we cited.

2.3. The authors write that they do not consider the rebound effect. In reality the rebound effect is likely to be more than $100 \%$ as health care is probably one of the least carbon intensive activities in the economy. If money is saved in health care they will most likely to spent in a more carbon intensive part of the economy. The authors also mentions the problems of longer life-spans etc, but does not seem to account for that. To make a real estimation would be much more demanding and would probably reach the conclusion that the emissions would increase. As it is now the calculation is misleading and should be omitted. RESPONSE: We do not agree that not estimating the effect of rebound and backfire on the system wide GHGE invalidates our calculations. In fact, most scientific papers estimating the effects of diet change on health and GHGE (e.g. Springmann et al. 2016; Tilman and Clark 2014) do not include analysis of the potential effects of rebound or of change in lifespans, because this is outside of the scope of the research. The contribution of this research, including our own, is to increase knowledge about the potential for reducing GHGE, not to model the way in which this potential would play out in the wider system.

2.4. The results cannot be reproduced and even evaluated as too little data is presented in the paper. Which LCA data is used for red meat, pulses etc, and the risk factors for health impact? This data must be presented in the paper or in an Appendix. Several methodological steps is also very briefly described as how waste from retail and households are handled or altered system boundaries. These part must be extended.

RESPONSE: We did describe these data and methods in detail in the submitted Online Resource, which it appears Reviewer 3 did not see. LCA data used were described in tables OR2 and OR4, and relative risks in table OR2. The methodology used to account for food waste is described in the Online Resource (section 1.5, paragraph following table OR1: "The purchased amount of food in SAD was based on "consumer weight" data from 2012 (USDA ERS 2014) 
which refer to the weight of the product as it is purchased at the retail level, i.e. after subtracting retail-level losses but before losses at the consumer level (Muth et al. 2011). The amount of food purchased in the HADs was quantified by assuming the same percentage of household food losses in each food category as in the SAD."

2.5. The discussion on GWP values is weak and uninformed. The author state that methane is important in the critical short term. This is not justified or explained, climate change is a long term challenge, what is critical in the near term? To use GWP20, mean that you ONLY consider the climate effect the next 20 years, and do not at all consider any effect thereafter. Therefore disregard melting of the Greenland ice cover or sea level rise, and the effect on coming generations. As the authors state this is a normative choice and not a scientific. Still, in the abstract they call GWP20 more "realistic".

RESPONSE: We agree that the term "realistic" is not a good choice, and have replaced it with "timely". However, we do think the 20 year GWP values are important to consider because they give a strong indication of how much warming can occurs over a short time period due to methane, and have added reference to (Scovronick et al. 2015) in support of using a 20-year GWP for methane. We have added section 1.6 in the Online Resource to address this in more detail, along with other comments related to GWP. However, we do not see this paper as a defense of using what is (for better or worse) a common set of metrics. Short term gains from reducing methane emissions could be critical for mitigating climate change, something we point out in the submitted ms. (section 1). We also included $100 \mathrm{GWP}$ values in our analysis, in order to provide a comparison with other research. Using the 20 year GWP for methane does not imply that we do not consider longer term climate effects of methane, which depends on emission rates. Rather, our intention is to focus on the potential for rapid response. Furthermore, because we emphasize that effective climate change mitigation in the short term is critical, our goal is to show that dietary choices can have an immediate effect. We do not, however, imply that diet is the only issue.

2.6. Even though the LCA studies use GWP100 of 21, this number is outdated, so there are no reason to report those values (GWP here accounted for only a few indirect effects). 34 is the reasonable number if GWP100 is used.

RESPONSE: We do not agree with Reviewer 3's point about providing results for a GWP of 21 for methane. Providing results for these three GWPs illustrates the sensitivity of estimates of diet impact on climate to GWP values. In addition, because so many studies in the past use inconsistent GWPs, we think it important to show that older studies and methodologies could come up with different values. Making this matter salient in the graphics is important as well, because many studies do not make adjustments when referring to previous literature. We have clarified these points in the new section 1.6 in Online Information.

2.7. The authors could have a discussion of different metrics and the effect of those, but need to carefully study the extensive literature on the subject first, starting for instance with Persson et al 2015 http://iopscience.iop.org/article/10.1088/1748-9326/10/3/034005/meta RESPONSE: While we did discuss this issue briefly in the main text, we agree this requires more attention, so we have added a discussion of climate impact metrics in the Online Resource (section 1.6). We found the suggested study by Persson et al. study valuable, and that it supports our current methodology. We considered the three GWP values for methane $(21,34$ and 86$)$ in in 
order 1) to be backwards compatible with older literature (GWP =21), 2) to have an up-to-date standard metric ( $\mathrm{GWP}=34)$, and 3) to emphasize the potential of rapid mitigation in response to behavioral change $(\mathrm{GWP}=86)$. Following Persson et al., we do not think it appropriate to use global temperature change potential (GTP), and have pointed this out in our addition to the Online Resource. In addition, GWP is by far the most commonly used indicator used in the LCA literature, we tried to remain consistent with the standard approaches in the field, with the exception of pointing out the potential impact of using the 20-year timeframe. However, considering the multiple issues in the use of GWP and GTP for methane would go well beyond the scope of our paper. We have clarified these points in the new section 1.6 in Online Information, and we have referenced this Online Information in the main text (sections 2.6 and 3.2).

2.8. The author's state on several occasions that diet shifts only require minimum investment and research etc. That is true, still authorities have tried to get people to eat healthy for decades with little success. Why would an abstract environmental threat make people to change eating habits, when their own health was not motivation enough? The authors should provide a more in-depth and nuance discussion about this, and discuss how the changes they are investigating could come about.

RESPONSE: See 1.18 above. This is an important topic, but beyond the scope of our paper. Like many other papers estimating the effects of diet change on climate and health (Springmann et al. 2016; Tilman and Clark 2014), our goal is to provide results that contribute to the discussion of the potential for diet change, and not to analyze how that change could come about. Our conclusion therefore highlights the potential for diet change to contribute to climate change mitigation, but does not discuss how that could happen.

2.9. The last sentence in the conclusion lacks any support in the paper as the implicit cost of behavioral changes has not been assessed.

RESPONSE: We agree that this statement would require further support, and have deleted it.

\section{References cited}

Micha R, Wallace SK, Mozaffarian D (2010) Red and Processed Meat Consumption and Risk of Incident Coronary Heart Disease, Stroke, and Diabetes Mellitus: A Systematic Review and Meta-Analysis Circulation 121:2271-2283 doi:10.1161/circulationaha.109.924977

Muth MK, Karns SA, Joy Nielsen S, Buzby JC, Wells HF (2011) Consumer-Level Food Loss Estimates and Their Use in the ERS Loss-Adjusted Food Availability Data. Technical Bulletin No. (TB-1927) USDA ERS,

Scovronick N, Dora C, Fletcher E, Haines A, Shindell D (2015) Reduce short-lived climate pollutants for multiple benefits The Lancet 386:e28-e31 doi:10.1016/S01406736(15)61043-1

Springmann M, Godfray HCJ, Rayner M, Scarborough P (2016) Analysis and valuation of the health and climate change cobenefits of dietary change Proceedings of the National Academy of Sciences doi:10.1073/pnas.1523119113 
Thoma G et al. (2013) Greenhouse gas emissions from milk production and consumption in the United States: A cradle-to-grave life cycle assessment circa 2008 International Dairy Journal 31, Supplement 1:S3-S14 doi:http://dx.doi.org/10.1016/j.idairyj.2012.08.013

Tilman D, Clark M (2014) Global diets link environmental sustainability and human health Nature 515:518-522 doi:10.1038/nature 13959 http://www.nature.com/nature/journal/v515/n7528/abs/nature13959.html supplementary-information

USDA, HHS (2010) Dietary Guidelines for Americans, 2010. 7th Edition. Washington, DC, U.S USDA ERS (2014) Food Availability (Per Capita) Data System. http://www.ers.usda.gov/dataproducts/food-availability-\%28per-capita\%29-data-system/.aspx - .U3qgyC-LnjE. Accessed 2014 August 14 


\section{References}

Aston LM, Smith JN, Powles JW (2012) Impact of a reduced red and processed meat dietary pattern on disease risks and greenhouse gas emissions in the UK: a modelling study BMJ Open 2 doi:10.1136/bmjopen-2012-001072

Bajželj B, Richards KS, Allwood JM, Smith P, Dennis JS, Curmi E, Gilligan CA (2014) Importance of food-demand management for climate mitigation Nature Climate Change 4:924-929 doi:10.1038/nclimate2353

Björklund A (2002) A Survey of approaches to improve reliability in LCA International Journal of LCA 7:64-72

CDC (2012) National Diabetes Fact Sheet, 2011 Centers for Disease Control and Prevention. http://www.cdc.gov/diabetes/pubs/factsheet11.htm. Accessed 2014 June 24

CDC (2014) National Diabetes Statistics Report: Estimates of Diabetes and Its Burden in the United States. U.S. Department of Health and Human Services; 2014. http://www.cdc.gov/diabetes/pubs/statsreport14.htm. Accessed 2014 June 24

CMS (2013) National Health Expenditure Projections 2012-2022. http://www.cms.gov/Research-Statistics-Data-and-Systems/Statistics-Trends-andReports/NationalHealthExpendData/Downloads/Proj2012.pdf. Accessed 2014 April 22

Doran-Browne N, Eckard R, Behrendt R, Kingwell R (2015) Nutrient density as a metric for comparing greenhouse gas emissions from food production Climatic Change 129:73-87 doi:10.1007/s10584-014-1316-8

Edenhofer O, R. Pichs-Madruga, Y. Sokona, E. Farahani, S. Kadner, K. Seyboth, A. Adler, I. Baum, S. Brunner, P. Eickemeier, B. Kriemann, J. Savolainen, S. Schlömer, C. von 
Stechow, T. Zwickel and J.C. Minx (ed) (2014) Climate Change 2014: Mitigation of Climate Change. Contribution of Working Group III to the Fifth Assessment Report of the Intergovernmental Panel on Climate Change. Cambridge University Press, Cambridge, United Kingdom and New York, NY, USA

EPA (2014a) Greenhouse Gas Equivalencies Calculator, Calculations and References. EPA. http://www2.epa.gov/energy/greenhouse-gas-equivalencies-calculator. Accessed 2014 June 15

EPA (2014b) Inventory of U.S. Greenhouse Gas Emissions and Sinks: 1990-2012. EPA. http://www.epa.gov/climatechange/ghgemissions/usinventoryreport.html. Accessed 2014 June 15

Friel S et al. (2009) Health and Climate Change 4 Public health benefits of strategies to reduce greenhouse-gas emissions: food and agriculture Lancet 374:2016-2025 doi:10.1016/s0140-6736(09)61753-0

Garnett T (2011) Where are the best opportunities for reducing greenhouse gas emissions in the food system (including the food chain)? Food Pol 36:S23-S32 doi:10.1016/j.foodpol.2010.10.010

GDI (2014) Economic Input-Output Life Cycle Assessment. GreenDesign Institute, Carnegie Mellon University. http://www.eiolca.net/index.html. Accessed 2014 January 15

Go AS et al. (2014) Heart Disease and Stroke Statistics—2014 Update: A Report From the American Heart Association Circulation 129:e28-e292 doi:10.1161/01.cir.0000441139.02102.80

Grotto D, Zied E (2010) The Standard American Diet and Its Relationship to the Health Status of Americans Nutrition in Clinical Practice 25:603-612 doi:10.1177/0884533610386234 
Hallström E, C-Kanyama, A., Börjesson, P. (2014) Environmental impact of dietary change: A systematic review $\mathbf{J}$ of Cleaner Production 91:1-11

Hallström E, Röös E, Börjesson P (2014) Sustainable meat consumption: A quantitative analysis of nutritional intake, greenhouse gas emissions and land use from a Swedish perspective Food Pol 47:81-90 doi:http://dx.doi.org/10.1016/j.foodpol.2014.04.002

Hansen J et al. (2013) Assessing "Dangerous Climate Change”: Required Reduction of Carbon Emissions to Protect Young People, Future Generations and Nature PLoS ONE 8:e81648 doi:10.1371/journal.pone.0081648

Heller MC, Keoleian GA (2015) Greenhouse Gas Emission Estimates of U.S. Dietary Choices and Food Loss Journal of Industrial Ecology 19:391-401 doi:10.1111/jiec.12174

Howarth RW (2014) A bridge to nowhere: methane emissions and the greenhouse gas footprint of natural gas Energy Science \& Engineering 2:47-60 doi:10.1002/ese3.35

IPCC (2013) Climate Change 2013: The Physical Science Basis. Working Group I contribution to the Fifth Assessment Report of the Intergovernmental Panel On Climate Change IPCC (2014) Climate Change 2014: Impacts, Adaptation, and Vulnerability. The Fifth Assessment Report (AR5). IPCC, Geneva

ISO (2006a) Environmental management. Life cycle assessment - Principles and framework. International Standard Organisation,, Geneve

ISO (2006b) Environmental management. Life cycle assessment - Requirements and guidelines. International Standard Organisation, Geneve

Jensen $\mathrm{H}$ et al. (2013) The importance of health co-benefits in macroeconomic assessments of UK Greenhouse Gas emission reduction strategies Climatic Change 121:223-237 doi:10.1007/s10584-013-0881-6 
Mäkiniemi J-P, Vainio A (2013) Moral intensity and climate-friendly food choices Appetite 66:54-61 doi:http://dx.doi.org/10.1016/j.appet.2013.01.026

Meier T, Christen O (2012) Environmental impacts of dietary recommendations and dietary styles: Germany as an example Environ Sci Technol 47:877-888

Nijdam D, Rood T, Westhoek H (2012) The price of protein: Review of land use and carbon footprints from life cycle assessments of animal food products and their substitutes Food Pol 37:760-770 doi:http://dx.doi.org/10.1016/j.foodpol.2012.08.002

Rogelj J, McCollum DL, Reisinger A, Meinshausen M, Riahi K (2013) Probabilistic cost estimates for climate change mitigation Nature 493:79-83 doi:10.1038/nature11787

Röös E, Josefine N (2013) Uncertainties and variations in the carbon footprint of livestock products. Rapport 063. Department of Energy and Technology, Swedish University of Agricultural Sciences, Uppsala, Sweden

Röös E, Karlsson H, Witthöft C, Sundberg C (2015) Evaluating the sustainability of dietscombining environmental and nutritional aspects Environmental Science \& Policy 47:157-166 doi:http://dx.doi.org/10.1016/j.envsci.2014.12.001

Rubinstein R, Kroese, D. (2007) Simulation and the Monte Carlo method. Wiley-Interscience, Saxe H, Larsen T, Mogensen L (2013) The global warming potential of two healthy Nordic diets compared with the average Danish diet Climatic Change 116:249-262 doi:10.1007/s10584-012-0495-4

Scarborough P, Allender S, Clarke D, Wickramasinghe K, Rayner M (2012) Modelling the health impact of environmentally sustainable dietary scenarios in the UK European journal of clinical nutrition 66:710-715 
Scarborough P, Appleby P, Mizdrak A, Briggs AM, Travis R, Bradbury K, Key T (2014a)

Dietary greenhouse gas emissions of meat-eaters, fish-eaters, vegetarians and vegans in the UK Climatic Change 125:179-192 doi:10.1007/s10584-014-1169-1

Scarborough P, Harrington RA, Mizdrak A, Zhou LM, Doherty A (2014b) The Preventable Risk Integrated ModEl and Its Use to Estimate the Health Impact of Public Health Policy Scenarios Scientifica 2014:21 doi:10.1155/2014/748750

SEER NCI (2014) Cancer Statistics. NCI. http://seer.cancer.gov/. Accessed 2014 April 22 Springmann M, Godfray HCJ, Rayner M, Scarborough P (2016) Analysis and valuation of the health and climate change cobenefits of dietary change Proceedings of the National Academy of Sciences doi:10.1073/pnas.1523119113

Stocker TF (2013) The Closing Door of Climate Targets Science 339:280-282 doi:10.1126/science. 1232468

Thoma $\mathrm{G}$ et al. (2013) Greenhouse gas emissions from milk production and consumption in the United States: A cradle-to-grave life cycle assessment circa 2008 International Dairy Journal 31, Supplement 1:S3-S14 doi:http://dx.doi.org/10.1016/j.idairyj.2012.08.013

Tilman D, Clark M (2014) Global diets link environmental sustainability and human health Nature advance online publication doi:10.1038/nature13959

Turner A et al. (2015) Estimating global and North American methane emissions with high spatial resolution using GOSAT satellite data

USDA (2010) Dietary Guidelines for Americans 2010

USDA, HHS (2010) Dietary Guidelines for Americans, 2010. 7th Edition. Washington, DC, U.S USDA, HHS (2015) Scientific Report of the 2015 Dietary Guidelines Advisory Committee. USDA, HHS, Washington, D.C. 
USDA ERS (2014) Food Availability (Per Capita) Data System. http://www.ers.usda.gov/dataproducts/food-availability-\%28per-capita\%29-data-system/.aspx - .U3qgyC-LnjE. Accessed 2014 August 14

van Dooren C, Marinussen M, Blonk H, Aiking H, Vellinga P (2014) Exploring dietary guidelines based on ecological and nutritional values: A comparison of six dietary patterns Food Pol 44:36-46 doi:http://dx.doi.org/10.1016/j.foodpol.2013.11.002

WCRF/AICR (2007) Food, Nutrition, Physical Activity, and the Prevention of Cancer: a Global Perspective. Washington DC: AICR

Westhoek H et al. (2014) Food choices, health and environment: Effects of cutting Europe's meat and dairy intake Global Environmental Change 26:196-205 doi:http://dx.doi.org/10.1016/j.gloenvcha.2014.02.004

WHO/FAO (2003) Diet, nutrition, and the prevention of chronic disease. Report of 164 a joint WHO/FAO Expert Consultation. Geneva, Switzerland 
1.a. SAD (standard American diet) (USDA)

1.c. HADs (healthy alternative diets):

- HAD-1 based on USDA recommendations. Decreased: RPM, refined grains; Increased: $F \& V$, B\&P, whole grains

- HAD-2 = HAD-1 with decreased RPM, increased B\&P

- HAD-3 = HAD-2 with decreased RPM, increased B\&P

\section{1.d. $\triangle$ (SAD-HADs)}

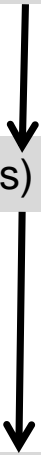

5. $\triangle$ GHGE-F in the food system for each food change for each HAD 1.b. Foods for which there were

(i) consistent dietary recommendations,

(ii) significant GHGE,

(iii) high quality $R R$ data for contribution to disease:

- Increased RR: RPM, refined grains

- Decreased RR: F\&V, whole grains

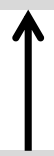

2. $\Delta$ disease prevalence: combined $\mathrm{RR}$ (RRcd) and $\mathrm{Cl}$ for all food $\Delta$ for each disease for each HAD

- colorectal cancer (CRC)

- type 2 diabetes (T2D)

- coronary heart disease (CHD)

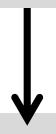

3. $\Delta$ health care costs in the US economy for each disease

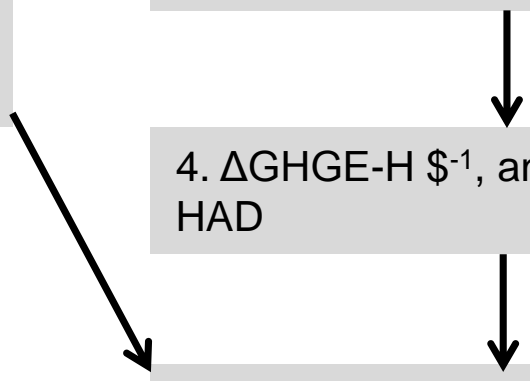

6. Net total $\triangle \mathrm{GHGE}$ from improved diet:

$\Delta$ GHGE-T $=(\Delta \mathrm{GHGE}-\mathrm{F}+\Delta \mathrm{GHGE}-$

H) 


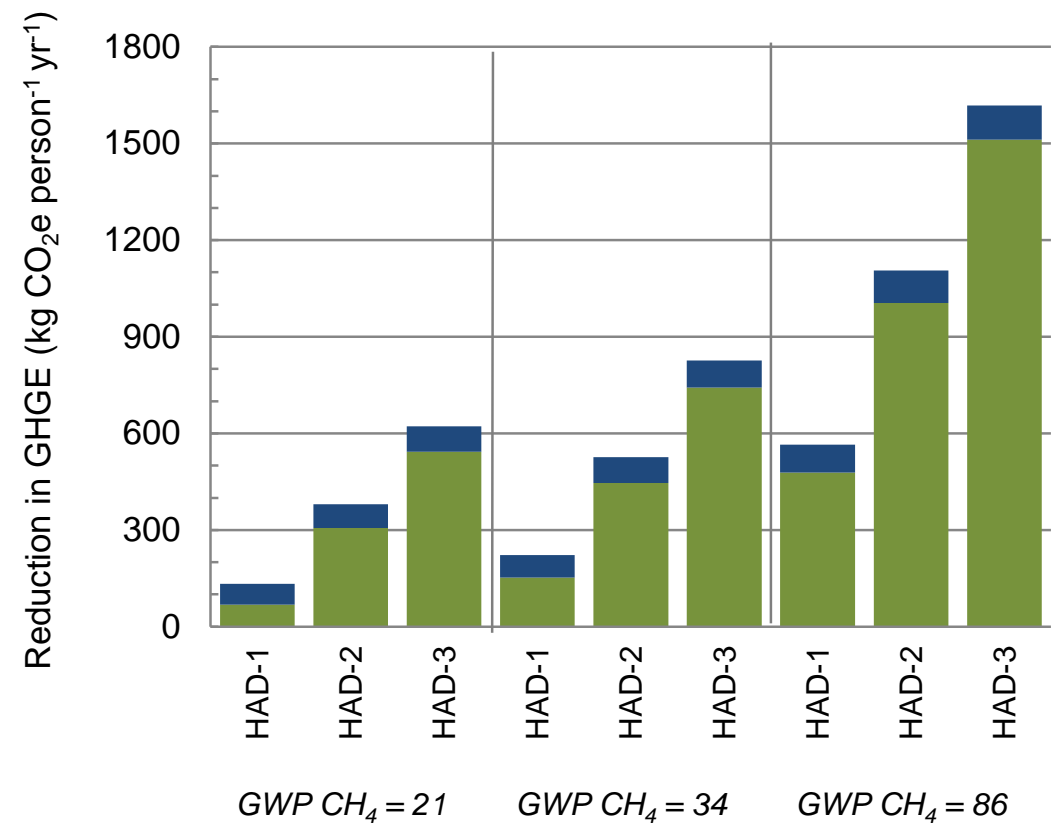

-Upstream nownstream 
Click here to access/download Supplementary Material OnlineResource.docx 


\section{Conflict of Interest}

Author Information. The authors declare no competing financial interests.

(2)

Author Information. The authors declare no competing financial interests.

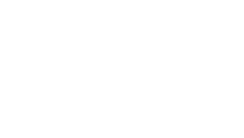

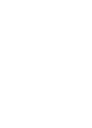

(1)

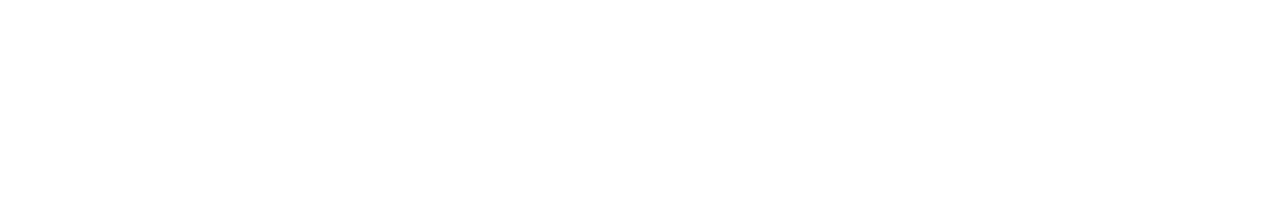

